\title{
ATTRACTING AND REPELLING POINT PAIRS FOR VECTOR FIELDS ON MANIFOLDS. I
}

\author{
GABRIELE MEYER
}

\begin{abstract}
Consider a compact, connected, $n$-dimensional, triangulable manifold $M$ without boundary, embedded in $\mathbf{R}^{n+1}$ and a continuous vector field on $M$, given as a map $f$ from $M$ to $S^{n}$ of degree not equal to 0 or $(-1)^{n+1}$. In this paper it is shown that there exists at least one pair of points $x, y \in M$ satisfying both $f(x)=-f(y)$ and $f(x)=\frac{x-y}{\|x-y\|}$. Geometrically, this means, that the points and the vectors lie on one straight line and the vector field is "repelling". Similarly, if the degree of $f$ is not equal to 0 or 1 , then there exists at least one "attracting" pair of points $x, y \in M$ satisfying both $f(x)=-f(y)$ and $f(x)=\frac{y-x}{\|y-x\|}$. The total multiplicities are $\frac{k^{\bullet}\left(k+(-1)^{n}\right)}{2}$ for repelling pairs and $\frac{k \cdot(k-1)}{2}$ for attracting pairs.

In the proof, we work with close simplicial approximations of the map $f$, using Simplicial, Singular and Čech Homology Theory, Künneth's Theorem, Hopf's Classification Theorem and the algebraic intersection number between two $n$-dimensional homology cycles in a $2 n$-dimensional space. In the case of repelling pairs, we intersect the graph of $f$ in $M \times S^{n}$ with the set of points $\left(x, \frac{x-y}{\|x-y\|}\right) \in M \times S^{n}$, where $x$ and $y$ satisfy that $f(x)=-f(y)$. In order to show that this set carries the homology $(k, k) \in H_{n}\left(M \times S^{n}, \mathbf{Z}\right)$, we study the set $A_{f} \equiv\{(x, y) \in M \times M \mid f(x)=-f(y)\}$ in a simplicial setting. Let $f_{j}$ be a close simplicial approximation of $f$. It can be shown, that $A_{f_{j}}$ is a homology cycle of dimension $n$ with a natural triangulation and a natural orientation and that $A_{f}$ and $A_{f_{j}}$ carry the same homology.
\end{abstract}

\section{INTRODUCTION}

Consider a compact, connected, $n$-dimensional, triangulable manifold $M$ without boundary, embedded in $\mathbf{R}^{n+1}$ and a continuous vector field on $M$, given as a map $f$ from $M$ to $S^{n}$ of degree not equal to 0 or $(-1)^{n+1}$. In this paper it is shown that there exists at least one pair of points $x, y \in M$ satisfying both $f(x)=-f(y)$ and $f(x)=\frac{x-y}{\|x-y\|}$. Geometrically, this means, that the points and the vectors lie on one straight line and the vector field is "repelling". Similarly, if the degree of $f$ is not equal to 0 or 1 , then there exists at least one "attracting" pair of points $x, y \in M$ satisfying both $f(x)=-f(y)$ and $f(x)=\frac{y-x}{\|y-x\|}$. The total multiplicities are $\frac{k \cdot\left(k+(-1)^{n}\right)}{2}$ for repelling pairs and $\frac{k \cdot(k-1)}{2}$ for attracting pairs.

This problem setting came up naturally, when D. W. Henderson and the author considered the following problem (see [2]): Is it possible to find appropri-

Received by the editors August 25, 1989.

1980 Mathematics Subject Classification (1985 Revision). Primary 55M25; Secondary 57Q55. 
ate notions of gradient vector field, gradient flows and Morse theory for a nonsmooth function $f \equiv \max \left\{f_{i} \mid i=1, \ldots, m\right\}$, where $f_{1}, \ldots, f_{m}$ are rational real-valued functions defined on an open subset $O$ of $\mathbf{R}^{n+2}$ ? In [2], we proved, that for every point $x$ in $O$ the function $f$ has one-sided directional derivatives; moreover, if at $x \in O f$ has a direction of descent, then, at $x \in O, f$ has a unique direction of steepest descent: $-\nabla f(x)$. We then proved that this noncontinuous vector field $-\nabla f$ has continuous solution curves everywhere in $O$ and that a version of Morse theory holds. In attempting to prove the uniqueness, of the solution curves, one naturally looks at the "regions" $A(a)$ in $O$ near $x$, where a certain subset of functions $f_{i_{1}}, \ldots, f_{i_{j}}, a=\left\{i_{1}, \ldots, i_{j}\right\}$, assumes the maximum and one tries to determine, in which of the regions-if there is a unique one-the solution curve to $-\nabla f$ through $x$ lies, immediately after passing through $x$. For that purpose, one takes $(n+1)$-dimensional, affine hyperplanes $H_{k}$ perpendicular to $-\nabla f(x)$ and approaching $x$ and considers the projection onto $H_{k}$ of $-\nabla f(y)$, where $y$ lies in one of the regions $A(i) \cap H_{k}$. If for infinitely many hyperplanes $H_{k}$ approaching $x$, there is an $A(i)$ such that on the boundary of $A(i) \cap H_{k}$ one obtains an attracting point pair, it is to be hoped that then there is a solution curve of $-\nabla f$ through $x$ in $A(i)$. If for infinitely many hyperplanes $H_{k}$ approaching $x$, there is an $A(i)$ such that on the boundary of $A(i) \cap H_{k}$ one obtains a repelling point pair, it is hoped that then the solution curve to $-\nabla f$ through $x$ bifurcates at $x$.

\section{THE MAIN THEOREM AND A PROOF OUTLINE}

The main result is

Theorem 1.1. Let $M$ be a compact, connected, triangulable, n-dimensional manifold without boundary embedded in $\mathbf{R}^{n+1}$ and let $f: M \rightarrow S^{n}$ be a continuous map of degree $k$. that

If $k \neq 0$ or $(-1)^{n+1}$, then there is at least one pair of points $x, y \in M$, such

1. $f(x)=-f(y)$,

2. $f(x)=\frac{x-y}{\|x-y\|}$,

and the total multiplicity of such point pairs is $\frac{k \cdot\left(k+(-1)^{n}\right)}{2}$.

Corollary 1.1. If $k \neq 0$ or 1 , then there is at least one pair of points $x, y \in M$, such that

1. $f(x)=-f(y)$,

2. $f(x)=\frac{y-x}{\|y-x\|}$,

and the total multiplicity of such point pairs is $\frac{k \cdot(k-1)}{2}$.

Proof of Corollary 1.1. Consider the map $f^{\prime} \equiv-f$, whose degree is $(-1)^{n+1}$. $\operatorname{deg}(f)$. Finding a pair of points $x_{i}, y_{i} \in M$, satisfying

(a1) $f^{\prime}\left(x_{i}\right)=-f^{\prime}\left(y_{i}\right)$,

(a2) $f^{\prime}\left(x_{i}\right)=\frac{y_{i}-x_{i}}{\left\|y_{i}-x_{i}\right\|}$

is equivalent to finding a pair of points $x_{i}, y_{i} \in M$, satisfying

(b1) $-f\left(x_{i}\right)=f\left(y_{i}\right)$,

(b2) $f\left(x_{i}\right)=\frac{x_{i}-y_{i}}{\left\|x_{i}-y_{i}\right\|}$. 
In case (b) we can apply Theorem 1.1 to obtain the multiplicity

$$
\frac{(-1)^{n+1} k \cdot\left((-1)^{n+1} \cdot k+(-1)^{n}\right)}{2}=\frac{k \cdot(k-1)}{2} .
$$

This number is not 0 , whenever $k \neq 0$ or $(-1)^{n+1}$.

Remark. Theorem 1.1 and Corollary 1.1 do not have gaps for the degrees 0 , $(-1)^{n+1}$ and 0,1 respectively: there are easy examples of functions with those degrees defined on $S^{n}$, for which there are no repelling or attracting pairs.

If $f \equiv$ constant, then the degree of $f$ is 0 and there are neither attracting nor repelling pairs.

If $f \equiv$ identity map, then the degree of $f$ is 1 and there are no attracting pairs.

If $f \equiv$ antipodal map, then the degree of $f$ is $(-1)^{n+1}$ and there are no repelling pairs.

Proof Outline for Theorem 1.1. We algebraically intersect the sets

$$
K_{f} \equiv\left\{\left(x, \frac{x-y}{\|x-y\|}\right) \in M \times S^{n} \mid f(x)=-f(y), x, y \in M\right\},
$$

and

$$
G_{f} \equiv\left\{(x, f(x)) \in M \times S^{n} \mid x \in M\right\},
$$

because any element in the intersection of these two sets automatically satisfies the conditions needed for a repelling pair. The algebraic intersection between the homology classes carried by $K_{f}$ and $G_{f}$ counts these occurrences with multiplicity. If the number is not 0 , then we can guarantee at least one repelling pair.

$G_{f}$, being the graph of $f$, can easily be shown to carry the homology

$$
(1, k) \in H_{n}\left(M \times S^{n}, \mathbf{Z}\right),
$$

and so the main effort is to show, that $K_{f}$ carries the homology

$$
(k, k) \in H_{n}\left(M \times S^{n}, \mathbf{Z}\right) .
$$

This will be done in several steps:

In $\S 2$, we will deal with the case of a particularly nice map $g_{0}: S^{n} \rightarrow S^{n}$ of degree $k$, where $K_{g_{0}}$ is the union of the graphs of $k$ functions of degree 1 ; this implies that $K_{g_{0}}$ carries the desired homology.

In $\S 3$, we introduce geodesically simplicial maps and state the key technical lemma:

Lemma 3.1. Let $f_{0}, f_{1}: T M \rightarrow G S^{n}$ be geodesically simplicial and noncollapsing maps of degree $k \neq 0$, then the following two sets

$$
\begin{aligned}
& A_{f_{0}} \equiv\left\{(x, y) \in M \times M \mid f_{0}(x)=-f_{0}(y)\right\} \quad \text { and } \\
& A_{f_{1}} \equiv\left\{(x, y) \in M \times M \mid f_{1}(x)=-f_{1}(y)\right\}
\end{aligned}
$$

can be triangulated and oriented naturally as n-dimensional homology cycles and an $(n+1)$-dimensional homology cobordism $A_{H}$ can be constructed which has $A_{f_{0}}$ and $A_{f_{1}}$ as its boundary.

In $\S 4$, we show that for the continuous function $f$ of Theorem 1.1, $A_{f}$ carries the same homology in $H_{n}(M \times M-\Delta, \mathbf{Z})$ as each one of a sequence of 
$A_{f_{i}}$ 's, where the $f_{i}$ 's are geodesically simplicial maps uniformly approaching $f$. The idea is to show that $A_{f}$ contains an inverse limit involving the $A_{f_{i}}$ 's.

In $\S 5$, we define the map

$$
\begin{aligned}
\theta: M \times M-\Delta & \rightarrow M \times S^{n} \\
(x, y) & \mapsto\left(x, \frac{x-y}{\|x-y\|}\right),
\end{aligned}
$$

which sends $A_{f}$ to $K_{f}$. Using the result of $\S 4$, we know that $A_{f}$ carries the same homology in $H_{n}(M \times M-\Delta, \mathbf{Z})$ as $A_{f_{0}}$, where $f_{0}$ is a close geodesically simplicial and noncollapsing approximation of the ideal map $g_{0} \circ r p$, where $r p$ is a very close geodesically simplicial and noncollapsing approximation of the radial projection from $M$ onto a geometric sphere $S_{0}^{n}$, sitting in the bounded complement of $M$ in $\mathbf{R}^{n+1}$ and $g_{0}$ is the ideal map defined on $S^{n}$ with the same degree as $f_{0}$.

We prove that

(a) $K_{f}$ carries the homology $\theta_{*}\left[A_{f_{0}}\right] \in H_{n}\left(M \times S^{n}, \mathbf{Z}\right)$ and that

(b) $K_{f_{0}}=\theta\left(A_{f_{0}}\right)$ carries the homology $(k, k) \in H_{n}\left(M \times S^{n}, \mathbf{Z}\right)$.

The latter is accomplished by reducing the problem $\left(M, f_{0}\right)$ to the case $\left(S^{n}, g_{0}\right)$, and this case has been dealt with in $\S 2$.

Thus, one obtains that $K_{f}$ carries the desired homology

$$
(k, k) \in H_{n}\left(M \times S^{n}, \mathbf{Z}\right) \text {. }
$$

Finally, we give a proof of Theorem 1.1, by intersecting $K_{f}=\theta\left(A_{f}\right)$ with the graph of $f, G_{f}$. This ends the proof outline.

\section{Motivation AND EXAMPLE}

We define a map $g_{0}$ on $S^{n}$, where the assertion of the theorem can be verified easily.

Lemma 2.1. There is a map $g_{0}: S^{n} \rightarrow S^{n}$ of degree $k \neq 0$, where the set

$$
K_{g_{0}} \equiv\left\{\left(x, \frac{x-y}{\|x-y\|}\right) \in S^{n} \times S^{n} \mid g_{0}(x)=g_{0}(x), x \in S^{n}\right\}
$$

carries the homology $(k, k) \in H_{n}\left(S^{n} \times S^{n}, \mathbf{Z}\right)$.

Moreover, there are exactly $\frac{k \cdot\left(k+(-1)^{n}\right)}{2}$ many pairs of points $x_{i}, y_{i} \in S^{n}$, satisfying both

1. $g_{0}\left(x_{i}\right)=-g_{0}\left(y_{i}\right)$,

2. $g_{0}\left(x_{i}\right)=\frac{x_{i}-y_{i}}{\left\|x_{i}-y_{i}\right\|}$.

Proof. Define $g_{0}$ inductively:

$n=1$ : Let $\theta \in[0,2 \pi), x \in S^{1}$ and $x=e^{i \theta}$, then define $g_{0}(x)=$ $e^{i(-1)^{n-1} k \theta}$. Suppose, that this initial $S^{1}=\left\{\left(x_{1}, x_{2}, 0, \ldots, 0\right) \in \mathbf{R}^{n+1} \mid x_{1}^{2}+x_{2}^{2}=\right.$ $1\}$. Extend $g_{0}$ to $S^{n}$ by successively suspending with the antipodal maps.

Now define maps $y_{j}: S^{n} \rightarrow S^{n}, j=1, \ldots, k$, also inductively

$n=1: \quad y_{j}: S^{1} \rightarrow S^{1}$, let $x=e^{i \theta}$. Define $y_{j}(x)=e^{i\left(\theta+\frac{(2 j-1) \pi}{k}\right)}$, then $y_{j}$ is a homeomorphism on $S^{1}$ and

$$
g_{0}\left(y_{j}(x)\right)=-g_{0}(x) .
$$

Inductively define $y_{j}$ on $S^{n}$ by suspending with the antipodal map. 
Then $A_{g_{0}} \equiv\left\{\left(x, y_{j}(x)\right) \mid x \in S^{n}, j=1, \ldots, k\right\}$ is the union of $k$ graphs of $y_{j}$ and is represented by $\left[A_{g_{0}}\right]=k \in H_{n}\left(S^{n} \times S^{n}-\Delta, \mathbf{Z}\right) \cong \mathbf{Z}$, where $\Delta$ is the diagonal in $S^{n} \times S^{n}$.

The vector $\frac{x-y_{j}(x)}{\left\|x-y_{j}(x)\right\|}$ can be homotoped to $x$, because it never equals $-x$. The set

$$
K_{g_{0}, j} \equiv\left\{\left(x, \frac{x-y_{j}(x)}{\left\|x-y_{j}(x)\right\|}\right) \mid x \in S^{n}\right\}
$$

is represented by $\left[K_{g_{0}, j}\right]=(1,1) \in H_{n}\left(S^{n} \times S^{n}, \mathbf{Z}\right)$. Thus $\left[K_{g_{0}}\right] \equiv\left[\bigcup_{j=1}^{k} K_{g_{0}, j}\right]$ $=(k, k) \in H_{n}\left(S^{n} \times S^{n}, \mathbf{Z}\right)$.

The intersection of the graph $G$ of $g_{0}$ and $K_{g_{0}}$ all lie in the plane determined by the initial $S^{1}$. The algebraic intersection number between $[G]=$ $\left(1, \operatorname{deg}\left(g_{0}\right)\right)=(1, k)$ and $\left[K_{g_{0}}\right]=(k, k)$ gives us the number of such occurrences: $k \cdot\left(k+(-1)^{n}\right)$. But this counts a pair $x, y$ twice, so we get exactly $\frac{k \cdot\left(k+(-1)^{n}\right)}{2}$ many repelling pairs $x, y$.

\section{THE KEY TECHNICAL LEMMA AND ITS REDUCTION TO THE SIMPLICIAL CASE}

In this section, we state and partly prove the key technical lemma, which says that any two geodesically simplicial and noncollapsing maps $f_{0}, f_{1}$ have homologous "pull-backs" $A_{f_{0}}$ and $A_{f_{1}}$. This is the key constructive idea of the whole proof, because the set $K_{f}$ can be obtained as the image of $A_{f}$ under a continuous function defined later on. This lemma will allow us to reduce to the case of a function which is much easier to deal with than $f$.

Definitions and set up for the technical lemma. Let $T M$ be a simplicial triangulation of $M$. Let $G S^{n}$ be a symmetric, geodesic triangulation of $S^{n}$. For reference about geodesic triangulations of the sphere, see [1].

Definition 3.1. A triangulation $G$ of $S^{n}$ is called symmetric if for every simplex $\sigma=x_{0} x_{1} \cdots x_{l} \in G$, the simplex $-\sigma=\left(-x_{0}\right)\left(-x_{1}\right) \cdots\left(-x_{l}\right) \in G$.

From $G S^{n}$ we construct a triangulated, symmetric, polyhedral sphere $\Sigma^{n}$ by taking as its vertices the vertices of $G S^{n}$ and as its $l$-simplices the convex hull in $\mathbf{R}^{n+1}$ of the vertices $x_{0} x_{1} \cdots x_{l}$, where $\sigma=x_{0} x_{1} \cdots x_{l}$ is a geodesic simplex in $G S^{n}$. Denote this triangulation of $\Sigma^{n}$ by $T \Sigma^{n}$.

Let $c p: \Sigma^{n} \rightarrow S^{n}$ be the centric projection.

Definition 3.2. The map $f: T M \rightarrow G S^{n}$ is geodesically simplicial, if there exists a simplicial map $f^{\text {simp }}: T M \rightarrow G \Sigma^{n}$, so that $f=c p \circ f^{\text {simp }}$.

Note that $A_{f}=A_{f^{\text {simp }}}$. We will take $f_{0}^{\text {simp }}$ to be $f_{0}$ and $f_{1}^{\text {simp }}$ to be $f_{1}$.

Definition 3.3. The map $f: K \rightarrow L$ is noncollapsing, if for every simplex $\sigma \in$ $K, f$ restricted to $|\sigma|$ is a homeomorphism.

The key technical result is

Lemma 3.1. Let $f_{0}, f_{1}: T M \rightarrow G S^{n}$ be geodesically simplicial and noncollapsing maps of degree $k \neq 0$, then the following two sets

$$
\begin{aligned}
& A_{f_{0}} \equiv\left\{(x, y) \in M \times M \mid f_{0}(x)=-f_{0}(y)\right\} \quad \text { and } \\
& A_{f_{1}} \equiv\left\{(x, y) \in M \times M \mid f_{1}(x)=-f_{1}(y)\right\}
\end{aligned}
$$


can be triangulated and oriented naturally as n-dimensional homology cycles and an $(n+1)$-dimensional homology cobordism $A_{H}$ can be constructed which has $A_{f_{0}}$ and $A_{f_{1}}$ as its boundary.

In the proof of Lemma 3.1 we need a suitable homotopy $H$, which is constructed in

Proposition 3.1. Let $f_{0}, f_{1}: T M \rightarrow T \Sigma^{n}$ be simplicial and noncollapsing, then there exists a simplicial map

$$
H: T(M \times I) \rightarrow T\left(\Sigma^{n} \times I\right),
$$

such that

1. $H$ is noncollapsing,

2. $H$ is level preserving,

3. $T\left(\Sigma^{n} \times I\right)$ is equivariant with respect to the antipodal map on $S^{n}$,

4. $H(x, 0)=\left(f_{0}(x), 0\right)$ and $H(x, 1)=\left(f_{1}(x), 1\right)$.

Proof. By Hopf's Theorem and the Simplicial Approximation Theorem, there is a simplicial homotopy $H: M \times I \rightarrow \Sigma^{n}$ with $H(x, 0)=f_{0}(x)$ and $H(x, 1)=$ $f_{1}(x)$. With appropriate subdivisions we can assume that $H$ is level preserving. Using general position we can take $H$ to be noncollapsing. If necessary we have to subdivide again to make $T\left(\Sigma^{n} \times I\right)$ equivariant with respect to the antipodal map on $S^{n}$.

The construction of the pull-back $A_{H}$ for $H$ involves a lot of checking, which is left to the reader:

$$
A_{H} \equiv\{(x, y, t) \in M \times M \times I \mid H(x, t)=(z, t) \& H(y, t)=(-z, t)\} .
$$

$A_{H}$ can be triangulated as a simplicial complex and has $A_{f_{0}}$ and $A_{f_{1}}$ as its boundary.

\section{THE REDUCTION FROM THE CONTINUOUS $f$ TO THE GEODESICALLY SIMPLICIAL AND NONCOLLAPSING $f_{i}$ VIA AN INVERSE LIMIT}

Consider the following subset of $(M \times M-\Delta) \times[m, \infty)$ :

$$
B_{m}^{\prime} \equiv\left\{(x, y, j) \in(M \times M-\Delta) \times[m, \infty) \mid j \in \mathbf{Z}, j \geq m \text { and }(x, y) \in A_{f_{j}}\right\} \text {. }
$$

Let $B_{m}$ be the closure of $B_{m}^{\prime}$ in $(M \times M-\Delta) \times[m, \infty]$, and let $i_{m+k, m}: B_{m+k} \hookrightarrow$ $B_{m}, k \geq 0$, be the inclusion map, then $\left(B_{m}, i_{m, m-1}\right)$ forms an inverse system with limit $B_{\infty}=\bigcap_{m=1}^{\infty} B_{m}$.

Lemma 4.1. $B_{\infty}$ in $(M \times M-\Delta) \times\{\infty\}$ carries the homology $z_{0}-\left[A_{f_{m}}\right]$, for all $m \in \mathbf{N}$, in $H_{n}((M \times M-\Delta), \mathbf{Z})$.

Proof. It can be checked that $A_{H_{m, m+1}}$ with boundary $A_{f_{m}} \cup A_{f_{m+1}}$ does not intersect the diagonal $\Delta \times[0,1]$ in $M \times M \times[0,1]$.

The following diagram commutes for all $m \in \mathbf{N}$ :

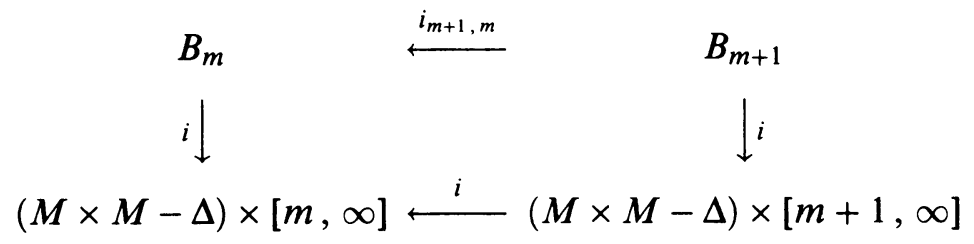


The bottom inclusion map induces the identity in homology, because $(M \times$ $M-\Delta) \times[m, \infty]$ can be deformation retracted to $(M \times M-\Delta) \times[m+1, \infty]$.

From the above we obtain an inverse system in homology:

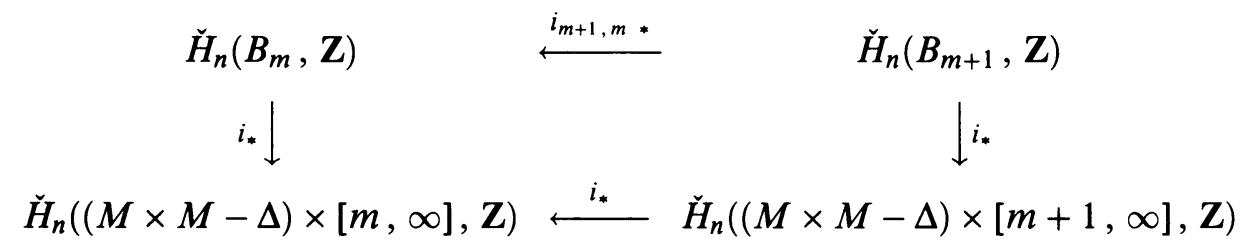

where $\breve{H}_{n}$ means Čech homology. By passing to the limit, we get by Theorem 5.8 (p. 195) in [6], that

$$
\begin{aligned}
\check{H}_{*}((M \times M-\Delta) \times[m, \infty], \mathbf{Z}) & =\check{H}_{*}((M \times M-\Delta) \times\{\infty\}, \mathbf{Z}) \\
& =\check{H}_{*}((M \times M-\Delta), \mathbf{Z}) \\
& =H_{*}((M \times M-\Delta), \mathbf{Z})
\end{aligned}
$$

here, the Čech homology groups are the same as the singular homology groups, because all the spaces are $2 n$-dimensional manifolds, which are paracompact and Hausdorff (see [3, p. 220]). By the same theorem in [6], we also get that

$$
\check{H}_{*}\left(B_{\infty}, \mathbf{Z}\right)=\lim _{\longleftarrow} \check{H}_{*}\left(B_{m}, \mathbf{Z}\right) .
$$

For each $m$, consider $\left\{i_{*} g \in \check{H}_{n}((M \times M-\Delta) \times[m, \infty], \mathbf{Z}) \mid g \in \check{H}_{n}\left(B_{m}, \mathbf{Z}\right)\right\}$.

The following diagram commutes, since all the maps are induced by inclusions:

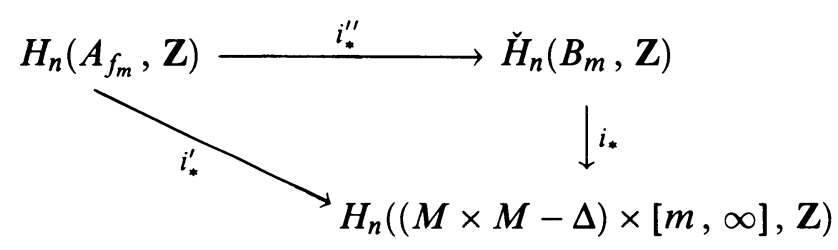

Thus $i_{*}^{\prime \prime}\left[A_{f_{m}}\right]$ is an element in $i_{*} \check{H}_{n}\left(B_{m}, \mathbf{Z}\right)$ and from Lemma 3.1, we know, that $\left[A_{f_{m}}\right]$ and $\left[A_{f_{m+1}}\right]$ are homologous in $H_{n}((M \times M-\Delta) \times[m, m+1], \mathbf{Z})$.

Thus

$$
i_{*}^{\prime}\left[A_{f_{1}}\right]=i_{*}^{\prime}\left[A_{f_{2}}\right]=\cdots=i_{*}^{\prime}\left[A_{f_{m}}\right]=\cdots .
$$

Denote this cycle in $H_{*}((M \times M-\Delta), Z)$ by $z_{0}$. So $z_{0}$ lies in

$$
\left\{i_{*} g \in H_{n}((M \times M-\Delta) \times[m, \infty], \mathbf{Z}) \mid g \in \check{H}_{n}\left(B_{m}, \mathbf{Z}\right),\right.
$$

for all $m$. By passing to the limit, we can conclude by Theorem 5.8 (p. 195) in [6], that

$$
z_{0} \in\left\{i_{*} g \mid g \in \check{H}_{n}\left(B_{\infty}, \mathbf{Z}\right)\right\} \subset H_{n}((M \times M-\Delta) \times\{\infty\}, \mathbf{Z}) .
$$

Thus $B_{\infty}$ carries the homology $z_{0}$ in $H_{n}((M \times M-\Delta), \mathbf{Z})$.

Lemma 4.2. $B_{\infty} \subset A_{f}$.

Proof. Let $(x, y, t) \in B_{\infty}$, then $(x, y, t)=\lim \left(x_{j}, y_{j}, t_{j}\right)$ of points in $A_{f_{j}}$. We have: $x=\lim x_{j}, y=\lim y_{j}$ in $M$ and $\infty=\lim t_{j}, t_{j} \in \mathbf{Z} \cup\{\infty\}$. Since the functions $f_{m}$ converge uniformly to $f$, for any convergent sequence of points $z_{l} \rightarrow z, z \in M$ :

$$
\lim _{l} \lim _{m} f_{m}\left(z_{l}\right)=\lim _{m} \lim _{l} f_{m}\left(z_{l}\right)=f(z) .
$$


In particular, this implies that

$$
f(x)=\lim f_{j}\left(x_{j}\right)=\lim \left(-f_{j}\left(y_{j}\right)\right)=-f(y),
$$

and thus $(x, y) \in A_{f}$.

Corollary 4.1. Af carries the homology

$$
z_{0}=\left[A_{f_{m}}\right] \in H_{n}((M \times M-\Delta), \mathbf{Z})
$$

and in fact, so does any other $A_{h}$, where $\operatorname{deg}(h)=\operatorname{deg}(f)$.

\section{THE HOMOLOGY CARRIED BY $K_{f}$ AND THE PROOF OF THEOREM 1.1}

Let $M$ be as in Theorem 1.1 and without loss of generality assume that the unit $n$-sphere $S_{0}^{n}$ sits inside the bounded complement of $M$. Let $r p: M \rightarrow S_{0}^{n}$ be a very close geodesically simplicial and noncollapsing approximation of the radial projection onto $S_{0}^{n}$. Finally, let $g_{0}: S^{n} \rightarrow S^{n}$ be the map defined in $\S 2$, so that $\operatorname{deg}\left(g_{0}\right)=\operatorname{deg}(f)$. Define $f_{0}=g_{0} \circ r p$. We want $r p$ to satisfy the following condition:

There is a homotopy $G_{1}: M \times I \rightarrow \mathbf{R}^{n+1}, G_{1}(x, 0)=x$, and $G_{1}(x, 1)=$ $r p(x) \in S_{0}^{n}$, such that if $x, y \in M, f_{0}(x)=-f_{0}(y)$, then for all $t \in[0,1]$, $G_{1}(x, t) \neq G_{1}(y, t)$. Clearly, such a map $r p$ and homotopy $G_{1}$ can be found. Then

(a) The map $f_{0} \equiv g_{0} \circ r p$ from $M$ to $S^{n}$ has the same degree as $f$.

(b) There exists a geodesically simplicial and noncollapsing map $f_{0}^{\prime}: T M \rightarrow$ $G S^{n}$ such that $A_{f_{0}}$ is an $n$-cycle homologous to $A_{f_{0}^{\prime}}$ in $H_{n}((M \times M-\Delta), \mathbf{Z})$.

Part (b) may require a subdivision of the geodesical triangulation $G S^{n}$ of $S^{n}$ as given in $\S 2$ and uses that $g_{0}$ is finite to one and open. (See Figure.)

Lemma 5.1. Let $f$ be a continuous map from $M$ to $S^{n}$, let $f_{0}^{\prime}$ be the map from $M$ to $S^{n}$ constructed above, and let

$$
\theta: M \times M-\Delta \rightarrow M \times S^{n} \text { be defined by }
$$

$$
(x, y) \mapsto\left(x, \frac{x-y}{\|x-y\|}\right),
$$

then $K_{f}=\theta\left(A_{f}\right)$ carries the homology $\theta_{*}\left(\left[A_{f_{0}^{\prime}}\right]\right) \in H_{n}\left(M \times S^{n}, \mathbf{Z}\right)$.

Proof. $\theta$ is well defined on $M \times M-\Delta$ and continuous. By Corollary 4.1, $A_{f}$ carries the homology $\left[A_{f_{0}^{\prime}}\right]$ in $H_{n}((M \times M-\Delta), \mathbf{Z})$. Thus, the image $K_{f}=\theta\left(A_{f}\right)$ carries the homology $\theta_{*}\left[A_{f_{0}^{\prime}}\right]$, where

$$
\theta_{*}: H_{n}((M \times M-\Delta), \mathbf{Z}) \rightarrow H_{n}\left(M \times S^{n}, \mathbf{Z}\right)
$$

is the homomorphism induced by $\theta$.

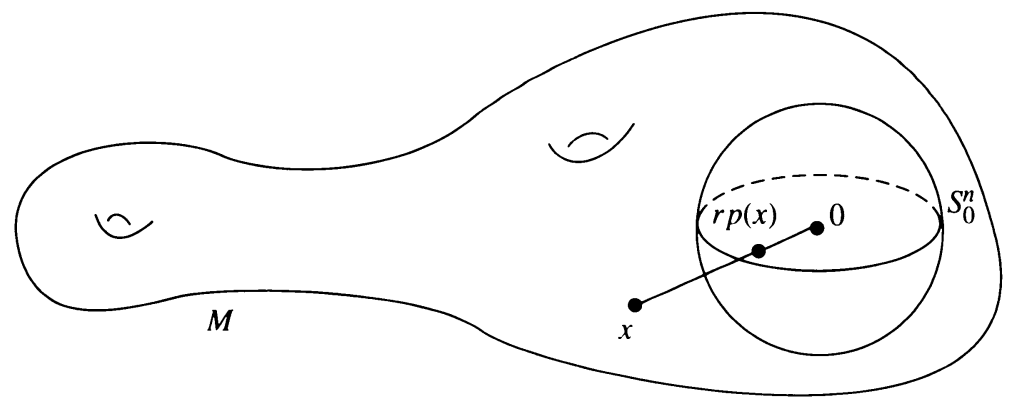

FIGURE 
Lemma 5.2. $K_{f_{0}}=\theta\left(A_{f_{0}}\right)$ carries the homology $(k, k)$ in $H_{n}\left(M \times S^{n}, \mathbf{Z}\right)$.

Proof. Using Künneth's formula we obtain $H_{n}\left(M \times S^{n}, \mathbf{Z}\right) \cong \mathbf{Z} \times \mathbf{Z}$. Take the homotopy $G_{1}$ retracting $M$ to $S_{0}^{n}$ as stated above, namely $G_{1}(x, 0)=x$ and $G_{1}(x, 1)=r p(x) \in S_{0}^{n}$, such that if $x, y \in M, f_{0}(x)=-f_{0}(y)$, then for all $t \in[0,1], G_{1}(x, t) \neq G_{1}(y, t)$. Next define a homotopy of the map $\theta$ :

$$
\begin{aligned}
G_{2}: A_{f_{0}} \times I & \rightarrow M \times S^{n} \\
(x, y, t) & \mapsto\left(x, \frac{G_{1}(x, t)-G_{1}(y, t)}{\left\|G_{1}(x, t)-G_{1}(y, t)\right\|}\right) .
\end{aligned}
$$

$G_{2}$ is well defined. Hence,

$$
\begin{aligned}
& G_{2}(x, y, 0)=\left(x, \frac{x-y}{\|x-y\|}\right)=\theta(x, y, 0), \\
& G_{2}(x, y, 1)=\left(x, \frac{r p(x)-r p(y)}{\|r p(x)-r p(y)\|}\right),
\end{aligned}
$$

and therefore $G_{2}\left(A_{f_{0}} \times\{0\}\right)=\theta\left(A_{f_{0}}\right)$ is homologous to $G_{2}\left(A_{f_{0}} \times\{1\}\right)$.

Consider the map

$$
\begin{gathered}
\xi: M \times M-\{(x, y) \in M \times M \mid y=\lambda \cdot x, \lambda>0\} \rightarrow S^{n}, \\
(x, y) \mapsto \frac{r p(x)-r p(y)}{\|r p(x)-r p(y)\|} .
\end{gathered}
$$

$\xi$ is well defined, because $r p(x) \neq r p(y), \xi$ is continuous and $\xi=G_{2}(\cdot, 1)$.

We know that $A_{f_{0}} \subset M \times M-\{(x, y) \in M \times M \mid y=\lambda \cdot x, \lambda>0\}$, because if $f_{0}(x)=-f_{0}(y)$, then $y \neq \lambda \cdot x, \lambda>0$. In terms of $x, \xi$ is homotopic to $r p(x)$ and in terms of $y, \xi$ is homotopic to $-r p(y)$. Thus $\xi$ has the degree $(-1)^{n+1}$ with respect to $y$. Therefore the map $\theta^{\prime} \equiv\left(\operatorname{id}_{M}, \xi\right)$ has degree $\left(1_{x},(-1)_{y}^{n+1}\right)$. The image of $\theta^{\prime}$ is contained in the set

$$
d \equiv\left\{(x, z) \in M \times S^{n} \mid z \neq-r p(x)\right\} .
$$

In particular, if $M=S^{n}$, denote the diagonal in $S^{n} \times S^{n}$ by $\Delta$ and the antidiagonal by $\Delta^{-}$; then $H_{n}\left(S^{n} \times S^{n}-\Delta, \mathbf{Z}\right) \cong \mathbf{Z}$ is generated by $\left[\Delta^{-}\right]$, $H_{n}\left(S^{n} \times S^{n}-\Delta^{-}, \mathbf{Z}\right) \cong \mathbf{Z}$ is generated by $[\Delta]$ and the map $\theta \equiv\left(\operatorname{id}_{S^{n}}, \xi\right)$ induces

$$
\theta_{*}: H_{n}\left(S^{n} \times S^{n}-\Delta, \mathbf{Z}\right) \rightarrow H_{n}\left(S^{n} \times S^{n}-\Delta^{-}, \mathbf{Z}\right),
$$

where $\theta_{*}\left[\Delta^{-}\right]=[\Delta]$.

Now consider the following diagram:

$$
\begin{array}{ccc}
M \times M-\{(x, y) \in M \times M \mid y=\lambda \cdot x, \lambda>0\} & \stackrel{\theta^{\prime}}{\longrightarrow} D=\left\{(x, z) \in M \times S^{n} \mid z \neq-r p(x)\right\} \\
(r p, r p) \downarrow & \downarrow^{\left(r p, \mathrm{id}_{S^{n}}\right)} \\
S^{n} \times S^{n}-\Delta & \stackrel{\theta}{\longrightarrow} & S^{n} \times S^{n}-\Delta^{-}
\end{array}
$$

This diagram commutes on the set level:

$$
\begin{aligned}
\left(r p, \operatorname{id}_{S^{n}}\right) \circ \theta^{\prime}(x, y) & =\left(r p, \operatorname{id}_{S^{n}}\right)\left(x, \frac{r p(x)-r p(y)}{\|r p(x)-r p(y)\|}\right) \\
& =\theta(r p(x), r p(y))=\theta \circ(r p, r p)(x, y) .
\end{aligned}
$$


Therefore it commutes in homology:

$$
\begin{array}{ccc}
H_{n}(M \times M-\{\cdots\}, \mathbf{Z}) \stackrel{\theta_{*}^{\prime}}{\longrightarrow} & H_{n}(D, \mathbf{Z}) \\
(r p, r p)_{*} \downarrow & & \downarrow\left(r p, \mathrm{id}_{S^{n}}\right) * \\
H_{n}\left(S^{n} \times S^{n}-\Delta, \mathbf{Z}\right) & \stackrel{\theta_{*}}{\longrightarrow} H_{n}\left(S^{n} \times S^{n}-\Delta^{-}, \mathbf{Z}\right)
\end{array}
$$

$D$ is homotopy equivalent to $\Delta^{\prime} \equiv\{(x, r p(x)) \mid x \in M\} \subset D$ :

$$
\begin{aligned}
& R: D \times I \rightarrow D \\
& (x, z, t) \mapsto\left(x, \frac{t \cdot r p(x)+(1-t) \cdot z}{\|t \cdot r p(x)+(1-t) \cdot z\|}\right) .
\end{aligned}
$$

$R$ is well defined, because $-r p(x) \neq z$, if $(x, z) \in D . R$ is a deformation retraction, because it is the identity on $\Delta^{\prime}$ for all $t \in I$. Therefore $H_{n}(D, \mathbf{Z})=$ $H_{n}\left(\Delta^{\prime}, \mathbf{Z}\right) \cong \mathbf{Z}$, generated by $\Delta^{\prime}$. Moreover $\theta_{*}\left[\Delta^{-}\right]=[\Delta],\left(r p, \operatorname{id}_{S^{n}}\right)_{*}\left[\Delta^{\prime}\right]=$ $[\Delta]$, thus they both induce the identity isomorphism on $\mathbf{Z}$. By construction $(r p, r p)\left(A_{f_{0}}\right)=A_{g_{0}}$ and $\left[A_{g_{0}}\right]=k \in H_{n}\left(S^{n} \times S^{n}-\Delta, \mathbf{Z}\right) \cong \mathbf{Z}$, by $\S 2$. Therefore we get

$$
\theta_{*}^{\prime}\left[A_{f_{0}}\right]=\left(r p, \operatorname{id}_{S^{n}}\right)_{*} \circ \theta_{*}^{\prime}\left[A_{f_{0}}\right]=\theta_{*} \circ(r p, r p)_{*}\left[A_{f_{0}}\right]=\theta_{*}\left[A_{g_{0}}\right]=k .
$$

Consider the homomorphism $i_{*}$, induced by the inclusion map $i: D \rightarrow M \times S^{n}$. $i_{*}$ sends the generator $\left[\Delta^{\prime}\right]$ of $H_{n}(D, \mathrm{Z})$ to the homology class generated by $1_{n} \otimes 1_{0}+1_{0} \otimes 1_{n}$, namely $(1,1)$. Therefore

$$
i_{*} \circ \theta_{*}^{\prime}\left[A_{f_{0}}\right]=(k, k) \in H_{n}\left(M \times S^{n}, \mathbf{Z}\right) .
$$

Thus $\theta^{\prime}\left(A_{f_{0}}\right)$ carries the homology $(k, k)$.

Corollary 5.1. $K_{f}=\theta\left(A_{f}\right)$ carries the homology $(k, k)$ in $H_{n}\left(M \times S^{n}, \mathbf{Z}\right)$.

Lemma 5.3. The algebraic intersection number of the homology classes $i_{1} \cdot\left(1_{n} \otimes\right.$ $\left.1_{0}\right)+j_{1} \cdot\left(1_{0} \otimes 1_{n}\right)$ and $i_{2} \cdot\left(1_{n} \otimes 1_{0}\right)+j_{2} \cdot\left(1_{0} \otimes 1_{n}\right)$ in $H_{n}\left(M^{n} \times S^{n}, \mathbf{Z}\right)$ is $i_{1} \cdot j_{2}+(-1)^{n} j_{1} \cdot i_{2}$.

Proof. See [3, p. 174].

Proof (of Theorem 1.1). Denote the graph of $f$ by

$$
G_{f} \equiv\left\{(x, f(x)) \in M \times S^{n} \mid x \in M\right\} .
$$

By hypothesis $f$ has degree $k \neq 0$. Therefore $\left[G_{f}\right]=(1, k) \in H_{n}\left(M \times S^{n}, \mathbf{Z}\right)$. The intersection number of $(k, k)$ with $\left[G_{f}\right]=(1, k)$ is $k \cdot\left(k+(-1)^{n}\right)$. Since $k \cdot\left(1+(-1)^{n} \cdot k\right) \neq 0$, we get at least one instance, where $f(x)=\frac{x-y}{\|x-y\|}$ and $f(x)=-f(y)$ hold simultaneously. However, the total multiplicity of such point pairs is $\frac{k \cdot\left(k+(-1)^{n}\right)}{2}$, since the algebraic intersection of $(k, k)$ and $(1, k)$ counts each pair $(x, y)$ at least twice.

\section{ACKNOWLEDGMENT}

I am very grateful to Professor David W. Henderson, my thesis advisor, who encouraged me to work on this topic, for the many helpful discussions and the frequent personal encouragement. In particular, I am indebted to him for his suggestions about $A_{f}$ containing the inverse limit of the sets $A_{f_{i}}$. I also want 
to thank Professor I. Bernstein, Professor M. Cohen, Professor R. Livesay and Professor P. Kahn from whom I received helpful suggestions and criticism. Last but not least I thank my husband Jin-yi Cai, whose questions led me to clarify and strengthen my intuitive ideas.

\section{REFERENCES}

1. M. Awartani and D. W. Henderson, Spaces of geodesic triangulations of the sphere, Trans. Amer. Math. Soc. 304 (1987), 721-732.

2. D. W. Henderson and G. E. Meyer, Gradient fields for the maximum of smooth functions, (submitted).

3. W. S. Massey, Singular homology theory, Graduate Texts in Math., No. 70, Springer-Verlag, New York, 1980.

4. C. P. Rourke and B. J. Sanderson, Introduction to piecewise linear topology, Springer Study Edition, Springer-Verlag, Berlin and Heidelberg, 1982.

5. E. H. Spanier, Algebraic topology, Springer-Verlag, New York, 1966.

6. A. H. Wallace, Algebraic topology: Homology and cohomology, Benjamin, New York, 1970.

28 Lake Lane, Princeton, New Jersey 08540 